\title{
A Hybrid Natural Transform Homotopy Perturbation Method for Solving Fractional Partial Differential Equations
}

\author{
Shehu Maitama \\ Department of Mathematics, Faculty of Science, Northwest University, Kano, Nigeria \\ Correspondence should be addressed to Shehu Maitama; smusman12@sci.just.edu.jo
}

Received 1 July 2016; Revised 23 August 2016; Accepted 4 September 2016

Academic Editor: Nasser-Eddine Tatar

Copyright (c) 2016 Shehu Maitama. This is an open access article distributed under the Creative Commons Attribution License, which permits unrestricted use, distribution, and reproduction in any medium, provided the original work is properly cited.

\begin{abstract}
A hybrid analytical method for solving linear and nonlinear fractional partial differential equations is presented. The proposed analytical approach is an elegant combination of the Natural Transform Method (NTM) and a well-known method, Homotopy Perturbation Method (HPM). In this analytical method, the fractional derivative is computed in Caputo sense and the nonlinear term is calculated using He's polynomial. The proposed analytical method reduces the computational size and avoids round-off errors. Exact solution of linear and nonlinear fractional partial differential equations is successfully obtained using the analytical method.
\end{abstract}

\section{Introduction}

The concept of fractional calculus which deals with derivatives and integrals of arbitrary orders [1] plays a significant role in many areas of physical science and engineering. Recently, there is a rapid development in the concept of fractional calculus and its applications [2-5]. The linear and nonlinear fractional differential equations are used to model significant problems in fluid mechanics, acoustic, electromagnetism, signal processing, analytical chemistry, biology, and many other areas of physical science and engineering [6]. In recent years, many analytical and numerical methods have been used to solve linear and nonlinear fractional differential equations such as Adomian Decomposition Method [710], Homotopy Analysis Method [11], Laplace Decomposition Method [12], Homotopy Perturbation Method [13, 14], and Yang Laplace transform [15]. Moreover, local fractional variational iteration method [16, 17], Cylindrical-Coordinate method [18], modified Laplace Decomposition Method [12, 19], and fractional complex transform method [20] are also applied to linear and nonlinear fractional partial differential equations. The asymptotic behavior of solutions of a weighted Cauchy-type nonlinear fractional partial differential equations is studied in [21-23].

It is evident that most of the existing methods have so many deficiencies such as unnecessary linearization, discretization of variables, transformation, or taking some restrictive assumptions.

In this paper, an analytical method called a Hybrid Natural Transform Homotopy Perturbation Method for solving linear and nonlinear fractional partial differential equations is introduced. The proposed analytical method is applied directly without any linearization, transformation, discretization of variables, and so on. The analytical method gives a series solution which converges rapidly to an exact or approximate solution with elegant computational terms. The nonlinear terms are elegantly computed using He's polynomials [24-26]. Exact solution of linear and nonlinear fractional partial differential equation is successfully obtained using the new analytical method. Thus, the Hybrid Natural Transform Homotopy Perturbation Method is a powerful mathematical method for solving linear and nonlinear fractional partial differential equations and is a refinement of the existing methods.

\section{Natural Transform}

In this section, the basic definition and properties of the Natural Transform are presented.

In the year 2008, Z. H. Khan and W. A. Khan [27] introduced an integral transform called $N$-transform and it 
TABLE 1: List of some special natural transforms.

\begin{tabular}{lc}
\hline Functional form & Natural transform form \\
\hline 1 & $\frac{1}{s}$ \\
$t$ & $\frac{u}{s^{2}}$ \\
$e^{a t}$ & $\frac{1}{s-a u}$ \\
$\frac{t^{n-1}}{(n-1) !}, n=1,2, \ldots$ & $\frac{u^{n-1}}{s^{n}}$ \\
$\sin (t)$ & $\frac{u}{s^{2}+u^{2}}$ \\
\hline
\end{tabular}

was recently renamed as the Natural Transform by Belgacem and Silambarasan $[28,29]$. In fact, based on personal communications and Internet records, CASM in LUMS and IOSS Forman Christian College in Lahore lectures in 2004 and 2005 and 3rd ICM4F COMSATs University conference side lectures in Islamabad in 2006 indicate that Belgacem shared and discussed various aspects of this transform, with the various attending audiences. The Natural Transform is an integral transform which is similar to Laplace transform [30] and Sumudu integral transform [31, 32]. It converges to Laplace transform when $u=1$ and Sumudu transform when $s=1$. Belgacem and Silambarasan [29, 33] have proposed a detailed theory and applications of the Natural Transform. Recently, Natural Transform and Adomian Decomposition Method are successfully combined and obtained an exact solution of linear and nonlinear partial differential equations [34-39]. More details about the Natural Transform and its applications can be seen in Wikipedia note about the Natural Transform [40].

Some useful Natural Transforms in this paper are presented in Table 1.

Definition 1. The Natural Transform of the function $v(t)>0$ and $v(t)=0$ for $t<0$ is defined over the set of functions:

$$
\begin{aligned}
A & =\left\{v(t): \exists M, \tau_{1}, \tau_{2}>0,|v(t)|<M e^{|t| / \tau_{j}}, \text { if } t\right. \\
& \left.\in(-1)^{j} \times[0, \infty), j=1,2, \ldots\right\},
\end{aligned}
$$

by the following integral:

$$
\begin{aligned}
& \mathbb{N}^{+}[v(t)]=V(s, u)=\frac{1}{u} \int_{0}^{\infty} e^{-s t / u} v(t) d t ; \\
& s>0, u>0 .
\end{aligned}
$$

And the inverse Natural Transform of the function $v(t)$ is defined by

$$
\mathbb{N}^{-1}[V(s, u)]=v(t)=\frac{1}{2 \pi i} \int_{\gamma-i \infty}^{\gamma+i \infty} e^{s t / u} V(s, u) d s
$$

where $s$ and $u$ are the Natural Transform variables [28, 29] and $\gamma$ is a real constant and the integral in (3) is taken along $s=\gamma$ in the complex plane $s=x+i y$.
Some properties of the Natural Transform Method are given below.

Property 1. Natural Transform of derivative: if $v^{n}(t)$ is the $n$th derivative of the function $v(t) \in A$ with respect to " $t$," then its Natural Transform is given by

$$
\mathbb{N}^{+}\left[v^{n}(t)\right]=\frac{s^{n}}{u^{n}} V(s, u)-\sum_{k=0}^{n-1} \frac{s^{n-(k+1)}}{u^{n-k}} v^{k}(0) .
$$

When $n=1,2$, and 3 , we have the following results:

$$
\begin{aligned}
\mathbb{N}^{+}\left[v^{\prime}(t)\right]= & \frac{s}{u} V(s, u)-\frac{1}{u} v(0), \\
\mathbb{N}^{+}\left[v^{\prime \prime}(t)\right]= & \frac{s^{2}}{u^{2}} V(s, u)-\frac{s}{u^{2}} v(0)-\frac{1}{u} v^{\prime}(0), \\
\mathbb{N}^{+}\left[v^{\prime \prime \prime}(t)\right]= & \frac{s^{3}}{u^{3}} V(s, u)-\frac{s^{2}}{u^{3}} v(0)-\frac{s}{u^{2}} v^{\prime}(0) \\
& -\frac{1}{u} v^{\prime \prime}(0) .
\end{aligned}
$$

Property 2. If $V(s, u)$ is the Natural Transform and $F(s)$ is the Laplace transform of the function $f(t) \in A$, then $\mathbb{N}^{+}[f(t)]=$ $V(s, u)=(1 / u) \int_{0}^{\infty} e^{-s t / u} f(t) d t=(1 / u) F(s / u)$.

Property 3. If $V(s, u)$ is the Natural Transform and $G(u)$ is the Sumudu transform of the function $v(t) \in A$, then $\mathbb{N}^{+}[v(t)]=$ $V(s, u)=(1 / s) \int_{0}^{\infty} e^{-t} v(u t / s) d t=(1 / s) G(u / s)$.

Property 4. If $\mathbb{N}^{+}[v(t)]=V(s, u)$, then $\mathbb{N}^{+}[v(\beta t)]=(1 / \beta) V(s /$ $\beta, u)$.

Property 5. The Natural Transform is a linear operator; that is, if $\alpha$ and $\beta$ are nonzero constants, then

$$
\begin{aligned}
\mathbb{N}^{+}[\alpha f(t) \pm \beta g(t)] & =\alpha \mathbb{N}^{+}[f(t)] \pm \beta \mathbb{N}^{+}[g(t)] \\
& =\alpha F^{+}(s, u) \pm \beta G^{+}(s, u) .
\end{aligned}
$$

Moreover, $F^{+}(s, u)$ and $G^{+}(s, u)$ are the Natural Transforms of functions $f(t)$ and $g(t)$, respectively.

\section{Basic Definitions of Fractional Calculus}

In this section, the basic definitions of fractional calculus are presented.

Definition 2. Function $f(t), t>0$, is said to be in the space $C_{\alpha}^{m}, m \in N \cup\{0\}$, if $f^{(m)} \in C_{\alpha}$.

Definition 3. A real function $f(t), t>0$, is said to be in the space $C_{\alpha} \alpha \in \mathbb{R}$ if there exists a real number $p(>\alpha)$ such that $f(t)=t^{p} f_{1}(t)$, where $f_{1}(t) \in C[0, \infty)$. Clearly $C_{\alpha} \subset C_{\beta}$ if $\beta \leq \alpha$. 
Definition 4. The left sided Riemann-Liouville fractional integral operator of order $\mu>0$, of a function $f(t) \in C_{\alpha}$, and $\alpha \geq-1$ is defined as $[19,41]$

$$
\begin{aligned}
& I^{\mu} f(t) \\
& \quad= \begin{cases}\frac{1}{\Gamma(\mu)} \int_{0}^{t}(t-\tau)^{\mu-1} f(\tau) d \tau, & \mu>0, t>0, \\
f(t), & \mu=0,\end{cases}
\end{aligned}
$$

where $\Gamma(\cdot)$ is the well-known Gamma function.

Definition 5. The left sided Caputo fractional derivative $f$, $f \in C_{1}^{m}, m \in \mathbb{N} \cup\{0\}$, is defined as $[1,5]$

$$
\begin{aligned}
D_{*}^{\mu} f(t) & \begin{cases}I^{m-\mu}\left[\frac{\partial^{m} f(t)}{\partial t^{m}}\right], & m-1<\mu<m, m \in \mathbb{N}, \\
\frac{\partial^{m} f(t)}{\partial t^{m}}, & \mu=m .\end{cases}
\end{aligned}
$$

Note that $[1,5]$

(i) $I_{t}^{\mu} f(t)=(1 / \Gamma(\mu)) \int_{0}^{t}\left(f(t) /(t-s)^{1-\mu}\right) d t, \mu>0, t>0$,

(ii) $D_{*}^{\mu} f(x, t)=I_{t}^{m-\mu}\left[\partial^{m} f(t) / \partial t^{m}\right], m-1<\mu \leq m$.

Definition 6. The Natural Transform of the Caputo fractional derivative is defined as

$$
\mathbb{N}^{+}\left[D_{t}^{n \alpha} v(t)\right]=\frac{s^{n \alpha}}{u^{n \alpha}} V(s, u)-\sum_{k=0}^{n-1} \frac{s^{n \alpha-(k+1)}}{u^{n \alpha-k}} v^{(k)}(0+),
$$

$((n-1) / n<\alpha \leq 1)$.

Definition 7. The series expansion defines a one-parameter Mittag-Leffler function as [1]

$$
E_{\alpha}(z)=\sum_{k=0}^{\infty} \frac{z^{k}}{\Gamma(\alpha k+1)}, \quad \alpha>0, z \in \mathbb{C} .
$$

\section{Analysis of the Method}

In this section, the basic idea of the Hybrid Natural Transform Homotopy Perturbation Method is clearly illustrated by the standard nonlinear fractional partial differential equation of the form

$$
D_{t}^{n \alpha} v(x, t)+M(v(x, t))+F(v(x, t))=g(x, t),
$$

subject to the initial condition

$$
v(x, 0)=f(x)
$$

where $F(v(x, t))$ represents the nonlinear terms, $D_{t}^{n \alpha}=$ $\partial^{n \alpha} / \partial t^{n \alpha}$ is the Caputo fractional derivative of function $v(t)$, $M(v(x, t))$ is the linear differential operator, and $g(x, t)$ is a source term.
Applying the Natural Transform to (11) subject to the given initial condition we get

$$
\begin{aligned}
V(x, s, u)= & \frac{1}{s} f(x)+\frac{u^{n \alpha}}{s^{n \alpha}} \mathbb{N}^{+}[g(x, t)] \\
& -\frac{u^{n \alpha}}{s^{n \alpha}} \mathbb{N}^{+}[M(v(x, t))+F(v(x, t))] .
\end{aligned}
$$

Taking the inverse Natural Transform of (13), we get

$$
\begin{aligned}
v(x, t) & \\
= & G(x, t) \\
& -\mathbb{N}^{-1}\left[\frac{u^{n \alpha}}{s^{n \alpha}} \mathbb{N}^{+}[M(v(x, t))+F(v(x, t))]\right],
\end{aligned}
$$

where $G(x, t)$ is a term arising from the source term and the prescribed initial condition.

Now we apply the Homotopy Perturbation Method:

$$
v(x, t)=\sum_{n=0}^{\infty} p^{n} v_{n}(x, t) .
$$

The nonlinear term $F(v(x, t))$ is decomposed as

$$
F(v(x, t))=\sum_{n=0}^{\infty} p^{n} H_{n}(v)
$$

where $H_{n}(v)$ is He's polynomial which is computed using the following formula:

$$
\begin{array}{r}
H_{n}\left(v_{1}, v_{2}, \ldots, v_{n}\right)=\frac{1}{n !} \frac{\partial^{n}}{\partial p^{n}}\left[F\left(\sum_{j=0}^{n} p^{j} v_{j}\right)\right]_{p=0}, \\
n=0,1,2, \ldots
\end{array}
$$

Substituting (16) and (15) into (14), we get

$$
\begin{aligned}
& \sum_{n=0}^{\infty} p^{n} v_{n}(x, t)=G(x, t)-p\left(\mathbb { N } ^ { - 1 } \left[\frac{u^{n \alpha}}{s^{n \alpha}}\right.\right. \\
& \left.\left.\cdot \mathbb{N}^{+}\left[\sum_{n=0}^{\infty} p^{n} M(v(x, t))+\sum_{n=0}^{\infty} p^{n} H_{n}(v)\right]\right]\right) .
\end{aligned}
$$

Using the coefficient of like powers of $p$ in (18), we obtained the following approximations:

$$
\begin{aligned}
p^{0} & : v_{0}(x, t)=G(x, t), \\
p^{1} & : v_{1}(x, t) \\
& =-\mathbb{N}^{-1}\left[\frac{u^{n \alpha}}{s^{n \alpha}} \mathbb{N}^{+}\left[M(v(x, t))+H_{0}(v)\right]\right],
\end{aligned}
$$




$$
\begin{aligned}
p^{2} & : v_{2}(x, t) \\
& =-\mathbb{N}^{-1}\left[\frac{u^{n \alpha}}{s^{n \alpha}} \mathbb{N}^{+}\left[M(v(x, t))+H_{1}(v)\right]\right], \\
p^{3} & : v_{3}(x, t) \\
& =-\mathbb{N}^{-1}\left[\frac{u^{n \alpha}}{s^{n \alpha}} \mathbb{N}^{+}\left[M(v(x, t))+H_{2}(v)\right]\right],
\end{aligned}
$$

and so on.

Hence, the series solution of (11)-(12) is given by

$$
v(x, t)=\lim _{N \rightarrow \infty} \sum_{n=0}^{N} v_{n}(x, t) .
$$

\section{Applications}

In this section, the application of the Hybrid Natural Transform Homotopy Perturbation Method to linear and nonlinear fractional partial differential equations is clearly demonstrated to show its simplicity and high accuracy.

Example 8. Consider the following fractional diffusion equation of the form

$$
\begin{aligned}
D_{t}^{\alpha} v+v_{x x}+v_{y y}+v_{z z}=0, & \\
& -\infty<x, y, z<\infty, t>0,
\end{aligned}
$$

subject to the initial condition

$$
v(x, y, z, 0)=e^{x+y+z}, \quad \alpha \in(0,1) .
$$

Applying the Natural Transform to (21) subject to the given initial condition, we get

$$
V(x, y, z, s, u)=\frac{e^{x+y+z}}{s}+\frac{u^{\alpha}}{s^{\alpha}} \mathbb{N}^{+}\left[v_{x x}+v_{y y}+v_{z z}\right]
$$

Taking the inverse Natural Transform of (23), we get

$$
\begin{aligned}
v(x, y, z, t)= & e^{x+y+z} \\
& +\mathbb{N}^{-1}\left[\frac{u^{\alpha}}{s^{\alpha}} \mathbb{N}^{+}\left[v_{x x}+v_{y y}+v_{z z}\right]\right] .
\end{aligned}
$$

Now we apply Homotopy Perturbation Method:

$$
v(x, y, z, t)=\sum_{n=0}^{\infty} p^{n} v_{n}(x, y, z, t) .
$$

Then (24) will become

$$
\begin{gathered}
\sum_{n=0}^{\infty} p^{n} v_{n}(x, y, z, t)=e^{x+y+z}-p\left(\mathbb { N } ^ { - 1 } \left[\frac{u^{\alpha}}{s^{\alpha}}\right.\right. \\
\left.\left.\cdot \mathbb{N}^{+}\left[\sum_{n=0}^{\infty} p^{n} v_{n x x}+\sum_{n=0}^{\infty} p^{n} v_{n y y}+\sum_{n=0}^{\infty} p^{n} v_{n z z}\right]\right]\right) .
\end{gathered}
$$

Using the coefficients of like powers of $p$ in (26), we obtained the following approximations:

$$
\begin{aligned}
p^{0} & : v_{0}(x, y, z, t)=e^{x+y+z}, \\
p^{1} & : v_{1}(x, y, z, t) \\
& =-\mathbb{N}^{-1}\left[\frac{u^{\alpha}}{s^{\alpha}} \mathbb{N}^{+}\left[v_{0 x x}+v_{0 y y}+v_{0 z z}\right]\right] \\
& =-\frac{e^{x+y+z} t^{\alpha}}{\Gamma(\alpha+1)}, \\
p^{2} & : v_{2}(x, y, z, t) \\
& =-\mathbb{N}^{-1}\left[\frac{u^{\alpha}}{s^{\alpha}} \mathbb{N}^{+}\left[v_{1 x x}+v_{1 y y}+v_{1 z z}\right]\right] \\
& =\frac{e^{x+y+z} t^{2 \alpha}}{\Gamma(2 \alpha+1)}, \\
p^{3} & : v_{3}(x, y, z, t) \\
& =-\mathbb{N}^{-1}\left[\frac{u^{\alpha}}{s^{\alpha}} \mathbb{N}^{+}\left[v_{2 x x}+v_{2 y y}+v_{2 z z}\right]\right] \\
& =-\frac{e^{x+y+z} t^{3 \alpha}}{\Gamma(3 \alpha+1)},
\end{aligned}
$$

and so on.

Then, the series solution of (21)-(22) is given by

$$
\begin{aligned}
& v(x, y, z, t)=\lim _{N \rightarrow \infty} \sum_{n=0}^{N} v_{n}(x, y, z, t)=v_{0}(x, y, z, t) \\
& +v_{1}(x, y, z, t)+v_{2}(x, y, z, t)+v_{3}(x, y, z, t)+\cdots \\
& =e^{x+y+z}\left(1-\frac{t^{\alpha}}{\Gamma(\alpha+1)}+\frac{t^{2 \alpha}}{\Gamma(2 \alpha+1)}\right. \\
& \left.-\frac{t^{3 \alpha}}{\Gamma(3 \alpha+1)}+\cdots\right)=e^{x+y+z}(1 \\
& \left.+\sum_{m=1}^{\infty} \frac{\left(-t^{\alpha}\right)^{m}}{\Gamma(m \alpha+1)}\right)=e^{x+y+z} E_{\alpha}\left(-t^{\alpha}\right) .
\end{aligned}
$$

When $\alpha=1$, the following result is obtained:

$$
\begin{aligned}
v(x, y, z, t)= & \lim _{N \rightarrow \infty} \sum_{n=0}^{N} v_{n}(x, y, z, t) \\
= & v_{0}(x, y, z, t)+v_{1}(x, y, z, t) \\
& +v_{2}(x, y, z, t)+v_{3}(x, y, z, t)+\cdots \\
= & e^{x+y+z}\left(1-\frac{t}{1 !}+\frac{t^{2}}{2 !}-\frac{t^{3}}{3 !}+\cdots\right) \\
= & e^{x+y+z-t},
\end{aligned}
$$

which is the exact solution of (21)-(22), when $\alpha=1$. 
Example 9. Consider the following coupled system of nonlinear fractional partial differential equations of the form

$$
\begin{gathered}
D_{t}^{\alpha} v-v_{x x}-2 v v_{x}+v_{x} w_{x}=0, \\
D_{t}^{\alpha} w-w_{x x}-2 w w_{x}+v_{x} w_{x}=0,
\end{gathered}
$$

subject to the initial conditions

$$
\begin{gathered}
v(x, 0)=\sin (x), \\
w(x, 0)=\sin (x) .
\end{gathered}
$$

Applying the Natural Transform to (30) subject to given initial conditions, we get

$$
\begin{aligned}
V(x, s, u)= & \frac{1}{s} \sin (x)+\frac{u^{\alpha}}{s^{\alpha}} \mathbb{N}^{+}\left[v_{x x}+2 v v_{x}-v_{x} w_{x}\right], \\
W(x, s, u)= & \frac{1}{s} \sin (x) \\
& +\frac{u^{\alpha}}{s^{\alpha}} \mathbb{N}^{+}\left[w_{x x}+2 w w_{x}-v_{x} w_{x}\right] .
\end{aligned}
$$

Taking the inverse Natural Transform of (32), we have

$$
\begin{aligned}
v(x, t)= & \sin (x) \\
& +\mathbb{N}^{-1}\left[\frac{u^{\alpha}}{s^{\alpha}} \mathbb{N}^{+}\left[v_{x x}+2 v v_{x}-v_{x} w_{x}\right]\right], \\
w(x, t)= & \sin (x) \\
& +\mathbb{N}^{-1}\left[\frac{u^{\alpha}}{s^{\alpha}} \mathbb{N}^{+}\left[w w_{x x}+2 w w_{x}-v_{x} w_{x}\right]\right] .
\end{aligned}
$$

Now we apply the Homotopy Perturbation Method:

$$
\begin{aligned}
& v(x, t)=\sum_{n=0}^{\infty} p^{n} v_{n}(x, t), \\
& w(x, t)=\sum_{n=0}^{\infty} p^{n} w_{n}(x, t) .
\end{aligned}
$$

Then (33) will become

$$
\begin{aligned}
v(x, t) & =\sin (x)+p\left(\mathbb { N } ^ { - 1 } \left[\frac{u^{\alpha}}{s^{\alpha}}\right.\right. \\
\cdot \mathbb{N}^{+} & {\left.\left.\left[\sum_{n=0}^{\infty} p^{n} v_{n x x}+2 \sum_{n=0}^{\infty} p^{n} H_{n}-\sum_{n=0}^{\infty} p^{n} H_{n}^{\prime \prime}\right]\right]\right), } \\
w(x, t) & =\sin (x)+p\left(\mathbb { N } ^ { - 1 } \left[\frac{u^{\alpha}}{s^{\alpha}}\right.\right. \\
\cdot \mathbb{N}^{+} & {\left.\left.\left[\sum_{n=0}^{\infty} p^{n} w_{n x x}+2 \sum_{n=0}^{\infty} p^{n} H_{n}^{\prime}-\sum_{n=0}^{\infty} p^{n} H_{n}^{\prime \prime}\right]\right]\right), }
\end{aligned}
$$

where $H_{n}, H_{n}^{\prime}$, and $H_{n}^{\prime \prime}$ are He's polynomials which represent the nonlinear terms $v v_{x}, w w_{x}$, and $v_{x} w_{x}$, respectively.
Some few components of He's polynomials of the nonlinear terms $v v_{x}, w w_{x}$, and $v_{x} w_{x}$ are given as follows:

$$
\begin{gathered}
H_{0}=v_{0} v_{0 x}, \\
H_{1}=v_{0} v_{1 x}+v_{1} v_{0 x}, \\
H_{2}=v_{0} v_{2 x}+v_{1} v_{1 x}+v_{2} v_{0 x}, \\
\vdots \\
H_{0}^{\prime}=w_{0} w_{0 x}, \\
H_{1}^{\prime}=w_{0} w_{1 x}+w_{1} w_{0 x}, \\
\vdots \\
w_{0} w_{2 x}+w_{1} w_{1 x}+w_{2} w_{0 x}, \\
H_{0}^{\prime \prime}=v_{0 x} w_{0 x}, \\
H_{1}^{\prime \prime}=v_{0 x} w_{1 x}+v_{1 x} w_{0 x}, \\
H_{2 x} w_{0 x}+v_{1 x} w_{1 x}+v_{0 x} w_{2 x},
\end{gathered}
$$

and so on.

Using the coefficients of the like powers of $p$ in (35), we obtained the following approximations:

$$
\begin{aligned}
p^{0} & : v_{0}(x, t)=\sin (x), \\
p^{1} & : v_{1}(x, t)=\mathbb{N}^{-1}\left[\frac{u^{\alpha}}{s^{\alpha}} \mathbb{N}^{+}\left[v_{0 x x}+2 H_{0}-H_{0}^{\prime \prime}\right]\right] \\
& =-\frac{\sin (x) t^{\alpha}}{\Gamma(\alpha+1)}, \\
p^{2} & : v_{2}(x, t)=\mathbb{N}^{-1}\left[\frac{u^{\alpha}}{s^{\alpha}} \mathbb{N}^{+}\left[v_{1 x x}+2 H_{1}-H_{1}^{\prime \prime}\right]\right] \\
& =\frac{\sin (x) t^{2 \alpha}}{\Gamma(2 \alpha+1)}, \\
p^{3} & : v_{3}(x, t)=\mathbb{N}^{-1}\left[\frac{u^{\alpha}}{s^{\alpha}} \mathbb{N}^{+}\left[v_{2 x x}+2 H_{2}-H_{2}^{\prime \prime}\right]\right] \\
& =-\frac{\sin (x) t^{3 \alpha}}{\Gamma(3 \alpha+1)},
\end{aligned}
$$

and so on.

Similarly,

$$
\begin{aligned}
& p^{0}: w_{0}(x, t)=\sin (x), \\
& p^{1}: w_{1}(x, t)=\mathbb{N}^{-1}\left[\frac{u^{\alpha}}{s^{\alpha}} \mathbb{N}^{+}\left[w_{0 x x}+2 H_{0}^{\prime}-H_{0}^{\prime \prime}\right]\right] \\
&=-\frac{\sin (x) t^{\alpha}}{\Gamma(\alpha+1)}
\end{aligned}
$$




$$
\begin{aligned}
p^{2} & : w_{2}(x, t)=\mathbb{N}^{-1}\left[\frac{u^{\alpha}}{s^{\alpha}} \mathbb{N}^{+}\left[w_{1 x x}+2 H_{1}^{\prime}-H_{1}^{\prime \prime}\right]\right] \\
& =\frac{\sin (x) t^{2 \alpha}}{\Gamma(2 \alpha+1)}, \\
p^{3} & : w_{3}(x, t)=\mathbb{N}^{-1}\left[\frac{u^{\alpha}}{s^{\alpha}} \mathbb{N}^{+}\left[w_{2 x x}+2 H_{2}^{\prime}-H_{2}^{\prime \prime}\right]\right] \\
& =-\frac{\sin (x) t^{3 \alpha}}{\Gamma(3 \alpha+1)},
\end{aligned}
$$

and so on.

Thus, the series solution of (30)-(31) is given by

$$
\begin{aligned}
& v(x, t)=\lim _{N \rightarrow \infty} \sum_{n=0}^{N} v_{n}(x, t)=v_{0}(x, t)+v_{1}(x, t) \\
& +v_{2}(x, t)+\cdots=\sin (x) \\
& \cdot\left(1-\frac{t^{\alpha}}{\Gamma(\alpha+1)}+\frac{t^{2 \alpha}}{\Gamma(2 \alpha+1)}-\frac{t^{3 \alpha}}{\Gamma(3 \alpha+1)}+\cdots\right) \\
& \quad=\sin (x)\left(1+\sum_{m=1}^{\infty} \frac{\left(-t^{\alpha}\right)^{m}}{\Gamma(m \alpha+1)}\right)=\sin (x) E_{\alpha}\left(-t^{\alpha}\right), \\
& w(x, t)=\lim _{N \rightarrow \infty} \sum_{n=0}^{N} w_{n}(x, t)=w_{0}(x, t)+w_{1}(x, t) \\
& \quad+w_{2}(x, t)+\cdots=\sin (x) \\
& \quad\left(1-\frac{t^{\alpha}}{\Gamma(\alpha+1)}+\frac{t^{2 \alpha}}{\Gamma(2 \alpha+1)}-\frac{t^{3 \alpha}}{\Gamma(3 \alpha+1)}+\cdots\right) \\
& \quad=\sin (x)\left(1+\sum_{m=1}^{\infty} \frac{\left(-t^{\alpha}\right)^{m}}{\Gamma(m \alpha+1)}\right)=\sin (x) E_{\alpha}\left(-t^{\alpha}\right) .
\end{aligned}
$$

Hence, the exact solutions of (30)-(31) are given by

$$
\begin{gathered}
v(x, t)=\sin (x) E_{\alpha}\left(-t^{\alpha}\right), \\
w(x, t)=\sin (x) E_{\alpha}\left(-t^{\alpha}\right) .
\end{gathered}
$$

When $\alpha=1$, the following result is obtained:

$$
\begin{aligned}
v(x, t) & =\lim _{N \rightarrow \infty} \sum_{n=0}^{N} v_{n}(x, t) \\
& =v_{0}(x, t)+v_{1}(x, t)+v_{2}(x, t)+\cdots \\
& =\sin (x)\left(1-t+\frac{t^{2}}{2 !}+\cdots\right)=e^{-t} \sin (x), \\
w(x, t) & =\lim _{N \rightarrow \infty} \sum_{n=0}^{N} w_{n}(x, t) \\
& =w_{0}(x, t)+w_{1}(x, t)+w_{2}(x, t)+\cdots \\
& =\sin (x)\left(1-t+\frac{t^{2}}{2 !}+\cdots\right)=e^{-t} \sin (x),
\end{aligned}
$$

which is the exact solution of (30)-(31), when $\alpha=1$.

\section{Conclusion}

In this paper, Natural Transform Method (NTM) and Homotopy Perturbation Method (HPM) are successfully combined to form a robust analytical method called a Hybrid Natural Transform Homotopy Perturbation Method for solving linear and nonlinear fractional partial differential equations. The analytical method gives a series solution which converges rapidly to an exact or approximate solution with elegant computational terms. In this analytical method, the fractional derivative is computed in Caputo sense, while the nonlinear term is calculated using He's polynomials. The analytical procedure is applied successfully and obtained an exact solution of linear and nonlinear fractional partial differential equations. The simplicity and high accuracy of the analytical method are clearly illustrated. Thus, the Hybrid Natural Transform Homotopy Perturbation Method is a powerful analytical method for solving linear and nonlinear fractional partial differential equations.

\section{Competing Interests}

The author declares that there is no conflict of interests regarding the publication of this article.

\section{References}

[1] I. Podlubny, Fractional differential equations, vol. 198 of Mathematics in Science and Engineering, Academic Press, San Diego, Calif, USA, 1999.

[2] K. S. Miller and B. Ross, An Introduction to the Fractional Calculus and Fractional Differential Equations, John Wiley \& Sons, New York, NY, USA, 2003.

[3] K. B. Oldham and J. Spanier, The Fractional Calculus, Academic Press, New York, NY, USA, 1974.

[4] K. Diethelm and N. J. Ford, "Analysis of fractional differential equations," Journal of Mathematical Analysis and Applications, vol. 265, no. 2, pp. 229-248, 2002.

[5] S. G. Samko, A. A. Kilbas, and O. I. Marichev, Fractional Integrals and Derivatives, Gordon and Breach, Yverdon, Switzerland, 1993.

[6] B. J. West, M. Bologna, and P. Grigolini, Physics of Fractal Operators, Institute for Nonlinear Science, Springer, New York, NY, USA, 2003.

[7] S. S. Ray, "Analytical solution for the space fractional diffusion equation by two-step Adomian decomposition method," Communications in Nonlinear Science and Numerical Simulation, vol. 14, no. 4, pp. 1295-1306, 2009.

[8] K. Abbaoui and Y. Cherruault, "New ideas for proving convergence of Adomian Decomposition Methods," Computers and Mathematics with Applications, vol. 32, pp. 103-108, 1995.

[9] S. Momani and Z. Odibat, "Analytical solution of a timefractional Navier-Stokes equation by Adomian decomposition method," Applied Mathematics and Computation, vol. 177, no. 2, pp. 488-494, 2006.

[10] H. Jafari and V. Daftardar-Gejji, "Solving linear and nonlinear fractional diffusion and wave equations by Adomian decomposition," Applied Mathematics and Computation, vol. 180, no. 2, pp. 488-497, 2006. 
[11] M. Ganjiani, "Solution of nonlinear fractional differential equations using homotopy analysis method," Applied Mathematical Modelling, vol. 34, no. 6, pp. 1634-1641, 2010.

[12] H. Jafari, C. M. Khalique, and M. Nazari, "Application of the Laplace decomposition method for solving linear and nonlinear fractional diffusion-wave equations," Applied Mathematics Letters, vol. 24, no. 11, pp. 1799-1805, 2011.

[13] P. K. Gupta and M. Singh, "Homotopy perturbation method for fractional Fornberg-Whitham equation," Computers \& Mathematics with Applications, vol. 61, no. 2, pp. 250-254, 2011.

[14] S. Momani and Z. Odibat, "Homotopy perturbation method for nonlinear partial differential equations of fractional order," Physics Letters. A, vol. 365, no. 5-6, pp. 345-350, 2007.

[15] Y.-Z. Zhang, A.-M. Yang, and Y. Long, "Initial boundary value problem for fractal heat equation in the semi-infinite region by yang-laplace transform," Thermal Science, vol. 18, no. 2, pp. 677681, 2014.

[16] X.-J. Yang and D. Baleanu, "Fractal heat conduction problem solved by local fractional variation iteration method," Thermal Science, vol. 17, no. 2, pp. 625-628, 2013.

[17] X.-J. Yang, D. Baleanu, Y. Khan, and S. T. Mohyud-Din, "Local fractional variational iteration method for diffusion and wave equations on Cantor sets," Romanian Journal of Physics, vol. 59, no. 1-2, pp. 36-48, 2014.

[18] X. J. Yang, H. M. Srivastava, J. H. He, and D. Baleanu, "Cantortype cylindrical-coordinate fractional derivatives," Proceedings of the Romanian Academy, Series A, vol. 14, pp. 127-133, 2013.

[19] S. Kumar, D. Kumar, S. Abbasbandy, and M. M. Rashidi, "Analytical solution of fractional Navier-Stokes equation by using modified Laplace decomposition method," Ain Shams Engineering Journal, vol. 5, no. 2, pp. 569-574, 2014.

[20] B. Ghazanfari and A. G. Ghazanfari, "Solving fractional nonlinear Schrodinger equation by fractional complex transform method," International Journal of Mathematical Modelling \& Computations, vol. 2, no. 4, pp. 277-281, 2012.

[21] K. M. Furati and N.-E. Tatar, "Longtime behavior for a nonlinear fractional model," Journal of Mathematical Analysis and Applications, vol. 332, no. 1, pp. 441-454, 2007.

[22] K. M. Furati and N.-E. Tatar, "Behavior of solutions for a weighted Cauchy type fractional problem," Journal of Fractional Calculus, vol. 28, pp. 23-42, 2005.

[23] K. M. Furati and N.-e. Tatar, "An existence result for a nonlocal fractional differential problem," Journal of Fractional Calculus, vol. 26, pp. 43-51, 2004.

[24] J.-H. He, "Homotopy perturbation technique," Computer Methods in Applied Mechanics and Engineering, vol. 178, no. 3-4, pp. 257-262, 1999.

[25] J. H. He, "Recent development of the homotopy perturbation method," Topological Methods in Nonlinear Analysis, vol. 31, pp. 205-209, 2008.

[26] J.-H. He, "The homotopy perturbation method for nonlinear oscillators with discontinuities," Applied Mathematics and Computation, vol. 151, no. 1, pp. 287-292, 2004.

[27] Z. H. Khan and W. A. Khan, "N-transform properties and applications," NUST Journal of Engineering Sciences, vol. 1, pp. 127133, 2008.

[28] F. B. M. Belgacem and R. Silambarasan, "Theory of the natural transform," Mathematics in Engineering, Science and Aerospace (MESA) Journal, vol. 3, no. 1, pp. 99-124, 2012.

[29] F. B. M. Belgacem and R. Silambarasan, "Advances in the natural transform," in Proceedings of the 9th International Conference on
Mathematical Problems in Engineering, Aerospace and Sciences (ICNPAA '12), vol. 1493 of AIP Conference Proceedings, pp. 106110, Vienna, Austria, July 2012.

[30] R. Murray, Spiegel. Theory and Problems of Laplace Transform, Schaum's Outline Series, McGraw-Hill, New York, NY, USA, 1965.

[31] F. B. Belgacem and A. Karaballi, "Sumudu transform fundamental properties investigations and applications," Journal of Applied Mathematics and Stochastic Analysis, vol. 2006, Article ID 91083, 23 pages, 2006.

[32] G. K. Watugala, "Sumudu transform-a new integral transform to solve differential equations and control engineering problems," Mathematical Engineering in Industry, vol. 6, no. 4, pp. 319-329, 1998.

[33] R. Silambarasan and F. B. M. Belgacem, "Application of the natural transform to Maxwell's equations," in Proceedings of the Progress in Electromagnetics Research Symposium Proceedings (PIERS '11), pp. 899-902, Suzhou, China, September 2011.

[34] M. Rawashdeh and S. Maitama, "Finding exact solutions of nonlinear PDEs using the natural decomposition method," Mathematical Methods in the Applied Sciences, 2016.

[35] S. Maitama and S. M. Kurawa, "An efficient technique for solving gas dynamics equation using the natural decomposition method," International Mathematical Forum, vol. 9, no. 24, pp. 1177-1190, 2014.

[36] S. Maitama, "Exact solution of equation governing the unsteady flow of a polytropic gas using the natural decomposition method," Applied Mathematical Sciences, vol. 8, no. 77, pp. 38093823, 2014.

[37] M. S. Rawashdeh and S. Maitama, "Solving PDEs using the natural decomposition method," Nonlinear Studies, vol. 23, no. 1, pp. 63-72, 2016.

[38] M. S. Rawashdeh and S. Maitama, "Solving nonlinear ordinary differential equations using the NDM," The Journal of Applied Analysis and Computation, vol. 5, no. 1, pp. 77-88, 2015.

[39] M. S. Rawashdeh and S. Maitama, "Solving coupled system of nonlinear PDE's using the natural decomposition method," International Journal of Pure and Applied Mathematics, vol. 92, no. 5, pp. 757-776, 2014.

[40] Wikipedia note about the Natural transform, August 2016, https://en.wikipedia.org/wiki/N-transform.

[41] Y. Luchko and R. Gorenflo, "An operational method for solving fractional differential equations with the Caputo derivatives," Acta Mathematica Vietnamica, vol. 24, no. 2, pp. 207-233, 1999. 


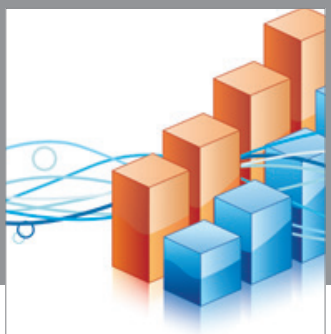

Advances in

Operations Research

vatem alat4

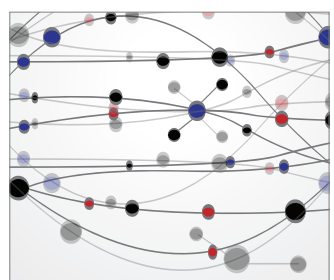

\section{The Scientific} World Journal
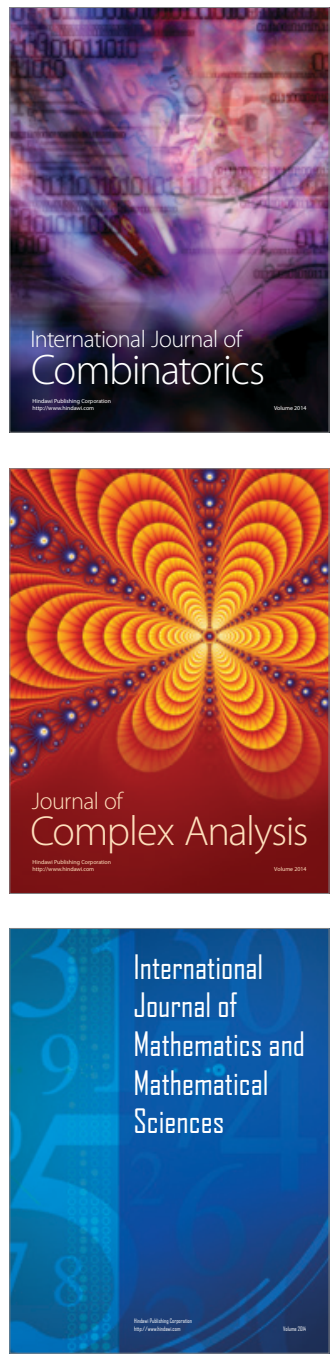
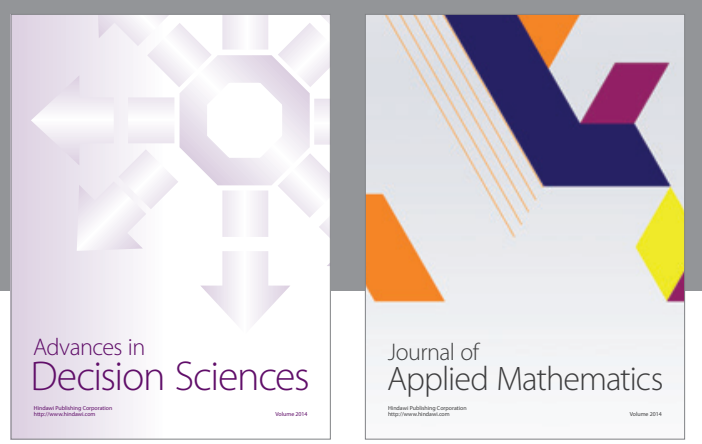

Algebra

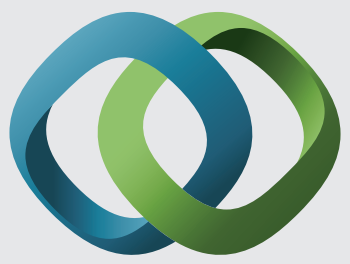

\section{Hindawi}

Submit your manuscripts at

http://www.hindawi.com
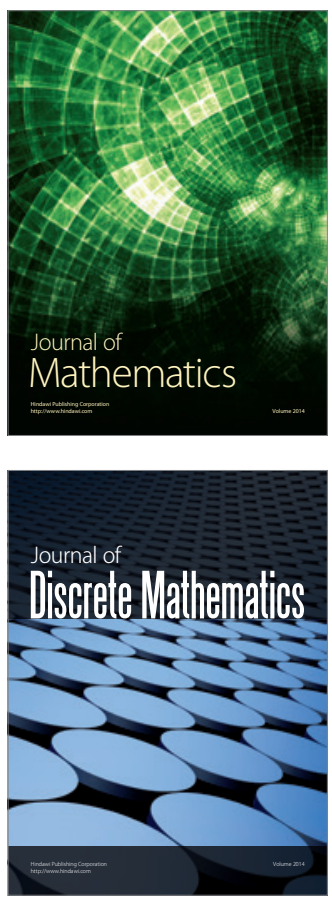

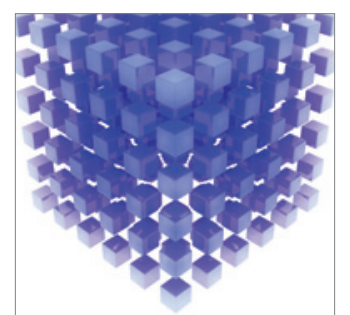

Mathematical Problems in Engineering
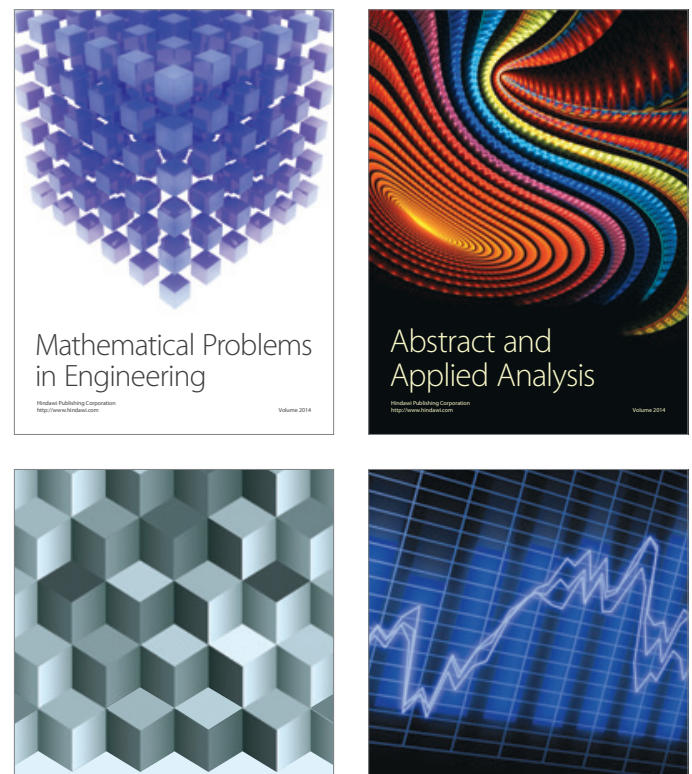

Journal of

Function Spaces

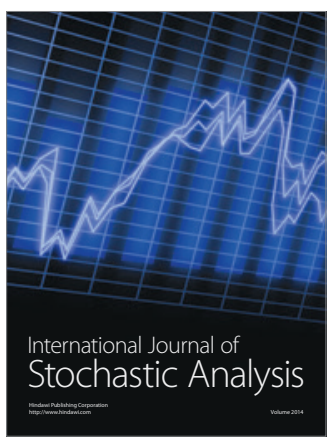

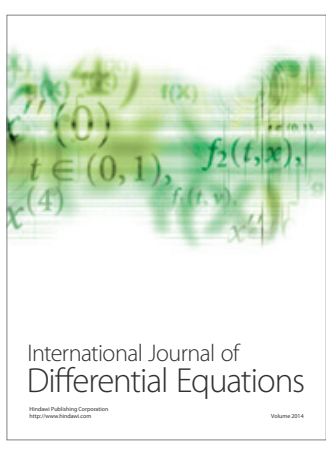
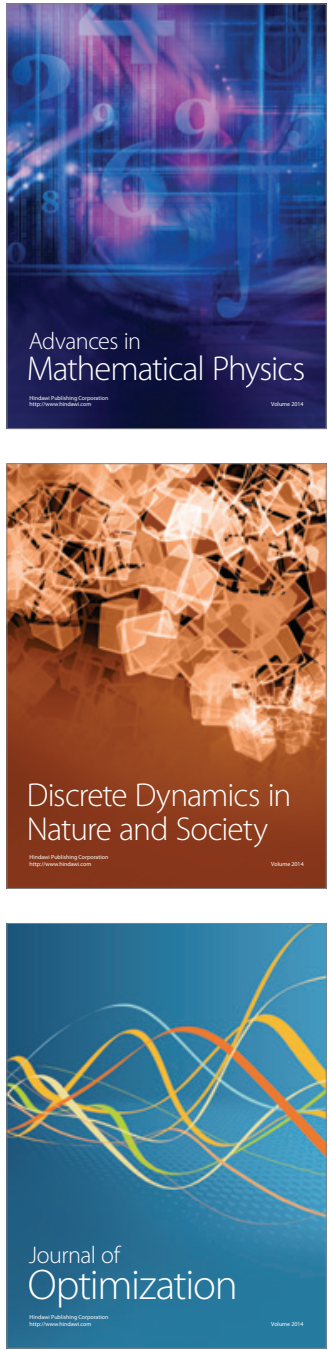